\title{
Complex measures and amplitudes, generalized stochastic processes and their applications to quantum mechanics
}

\author{
S K Srinivasan $\dagger$ and E C G Sudarshan \\ † Department of Mathematics, Indian Institute of Technology, Madras 600036, India \\ $\ddagger$ Center for Particle Physics and Department of Physics, University of Texas, Austin, TX 78712, \\ USA
}

Received 8 June 1992, in final form 15 April 1993

\begin{abstract}
Complex measure theory is used to widen the scope of the study of stochastic processes and it is shown how, with such an extension, the physical concepts of superposition and diffraction follow automatically. The Dirac-Feynman path integral formalism is seen as a natural development. Several generic Markov processes are studied when extended to complex measures. The role of conditional expectations in this framework as propagating amplitudes is brought out with special reference to the Huyghens' princple. Diffusion is studied in this extended formalism and the context in which the Schrödinger or Dirac equation can be derived is stated. The Hamiltonian evolution and decay of correlations require complex measures which are boundary values of analytic functions.
\end{abstract}

\section{Introduction}

The object of this contribution is to study the properties of an indexed family of complex measures. The motivation stems from the basic postulates of quantum mechanics that are currently in vogue. There are three apparently distinct ingredients contained in the postulates of quantum mechanics: they are, respectively, the uncertainty relations, Huyghens' principle and the algorithms that tell us how to go from a classical to a quantum system. In recent times the path integral formalism has become popular in view of its direct connection to probability theory with all the advantages of a collection of tools available for calculations. It is sad that these various developments are disjoint and ad hoc to a certain extent. Admittedly in the early formulation of quantum mechanics, the $\Psi$ function served as a mental block preventing further understanding in as much as only the squared moduli of $\Psi$ functions have a probabilistic interpretation. J von Neumann, to whom we owe our present set of postulates, was inspired very much by the science of classical statistical mechanics. In a certain sense the set of postulates is a kind of compromise that has led to a rather artificial postulational structure.

If we look at the problem afresh and interpret the two-slit experiment and other associated phenomena, we will be led naturally to the idea that the normal framework - of probability which deals with non-negative measures is not sufficient to describe quantum mechanics and that complex measures (which are amenable to both superposition and interference) are perhaps the best structure in terms of which problems of basic physics can be formulated and discussed. It is precisely such an idea that we pursue in this paper.

Some earlier attempts to use generalized phase-space densities is due to Wigner (1932) and the dynamics of such a generalized phase-space density was worked out by Moyal 
(1949) (see also Baker 1958, Jordan and Sudarshan 1961 and Gudder 1990). But in these cases these distributions are bilinear in the $\Psi$ function; and linear complex superpositions of densities is not a suitable density.

The plan of this paper is as follows. In section 2 we present salient features of the standard complex measure theory and introduce the generalized stochastic process as an indexed family of complex measures. We then introduce in section 3 the notion of complex Markov processes and study such Markov processes and deal with the major problem of identification of the Feynman path integral in the context of quantum mechanics of a single particle. We show explicitly that in the non-relativistic realm the Schrödinger equation a la path integral is a consequence of the Markov structure provided that the theory is complete. In section 4 we present some simple processes and indicate the potential of the current approach. The role of the conditional complex expectation as a propagating amplitude is explored in section 5 with a review of Huyghen's principle in the following section. Random diffusion with a complex measure is studied in section 7 . In section 8 the derivation of the Schrödinger and Dirac equations are outlined. Hamiltonian evolutions and the decay of correlations are studied in section 9 and the usefulness of analytic complex measures brought out in section 10. A brief summary is given in the concluding discussion in section 11 .

\section{Complex measures and generalized stochastic processes}

In measure theory we generally start with completely additive set functions which lead to signed measures (see e.g. Pitt 1968). The Hahn-Jordan decomposition enables us to deal with non-negative measures exclusively, the theorems that are proved for such measures being capable of extension to signed measures with trivial modifications. It is a standard exercise in measure theory to extend the concept to complex measures too (see e.g. Halmos 1950 p 120, Ash 1972). Thus in general a complex measure $\lambda$ can be defined for any set $A \in \mathcal{B}$ by

$$
\lambda(A)=\lambda_{1}(A)+i \lambda_{2}(A)
$$

where $\lambda_{1}$ and $\lambda_{2}$ are two finite signed measures on the measurable space $(\Omega, \mathcal{B})$. We now set (for reasons that will become obvious presently)

$$
\lambda(\Omega)=1
$$

and also insist $|\lambda(A)| \leqslant 1$ for any $A \in \mathcal{B}$. It is also a standard result that it is indeed possible to define an induced non-negative measure $|\lambda|$ called mod $\lambda$ by

$$
|\lambda|(A)=\sup \left|\int_{A} f d \lambda\right|
$$

where $f$ is any measurable complex function such that $|f| \leqslant 1$, the supreme being over all such functions $f$. We will have occasion to resort to this measure.

We now introduce the notion of a random variable. This is best done following Pitt, by choosing $\Omega=\mathcal{R}$ (the set of reals). If we wish to have finitely many random variables $\left(x_{1}, x_{2} \cdots x_{n}\right)$, we make copies of $\Omega$ and introduce $\Omega=\Omega_{1} \otimes \Omega_{2} \otimes \Omega_{3} \cdots \otimes \Omega_{n}$. The probability distribution $\mu(x)$ is called the joint distribution of $\left(x_{1}, x_{2}, \ldots x_{n}\right)$; we can consider the sets $X_{1}$ of $\Omega$ for which $X_{1} \otimes \Omega_{2} \otimes \ldots \otimes \Omega_{n}$ is measurable and we are naturally led to the measure

$$
\mu_{1}\left(X_{1}\right)=\mu\left(X_{1} \otimes \Omega_{2} \otimes \ldots \otimes \Omega_{n}\right) .
$$


The special case when

$$
\mu\left(x_{1} \otimes x_{2} \otimes \ldots \otimes x_{n}\right)=\mu_{1}\left(x_{1}\right) \mu_{2}\left(x_{2}\right) \ldots \mu_{n}\left(x_{n}\right)
$$

holds corresponds to the case when the random variables $x_{1}, x_{2}, \ldots, x_{n}$ are independent.

Let us now consider the case of a single random variable. We shall deal with the important case when the measure is absolutely continuous; then we can write

$$
\lambda(\mathrm{d} x)=\left[f_{1}(x)+i f_{2}(x)\right] \mathrm{d} x .
$$

We have

$$
\int\left(f_{1}+\mathrm{i} f_{2}\right) \mathrm{d} x=1
$$

or

$$
\int f_{1} \mathrm{~d} x=1 \quad \int f_{2} \mathrm{~d} x=0
$$

In view of the complex nature of the measure, we should be reconciled to the situation that the expectation of the random variable will in general be a complex number.

If we set the random variable as $\zeta$, the expectation of $\zeta$ may be written as

$$
E[\zeta]=\int \zeta\left(f_{1}+\mathrm{i} f_{2}\right) \mathrm{d} \zeta=\alpha+\mathrm{i} \beta
$$

Let

$$
\eta=\zeta-\alpha-\mathbf{i} \beta
$$

be a new random variable where $\eta$ is generated by the measurable space $\left(\Omega_{\beta}, F_{\beta}\right)$ and $\Omega_{\beta}$ is the set $\{x-\mathrm{i} \beta, x$ real $\}$ for fixed $\beta$. We then have

$$
E[\eta]=0
$$

and in this case we define the spread $\delta \eta$ or $\delta \zeta$ as

$$
\delta \zeta=\delta \eta=\int|x-\alpha-\mathrm{i} \beta|^{2}\left|f_{1}+\mathrm{i} f_{2}\right| \mathrm{d} x=\sigma^{2} .
$$

In other words, the spread is defined to be the expected value of the square of the displaced random variable with reference to the mod measure.

In terms of complex measure as probability we can deal with

$$
\operatorname{Pr}\{|\zeta-\alpha-i \beta|>\epsilon\}
$$

which is indeed a complex number. That such a probability can be defined in an appropriate measure space follows from the remarks above. We can, for simplicity, set

$$
\int x f_{2}(x) \mathrm{d} x=0
$$

without loss of generality. Then we have

$$
\begin{aligned}
|\operatorname{Pr}\{|\zeta-\alpha-\mathrm{i} \beta|>\epsilon\}| & =\int_{|x-\alpha|>\epsilon}\left|f_{1}+\mathrm{i} f_{2}\right| \mathrm{d} x \\
& \leqslant \epsilon^{-2} \int(x-\alpha)^{2}\left|f_{1}+\mathrm{i} f_{2}\right| \mathrm{d} x \\
& =\epsilon^{-2} \sigma^{2} .
\end{aligned}
$$


The bound given by (2.14) can be a multiple of the variance of the random variable only in limited context. This makes it rather difficult to interpret (2.14) as some kind of a Chebycheff bound. If $\beta$ does not vanish, we indeed have

$$
\operatorname{Pr}\left\{|\zeta-\alpha-i \beta| \leqslant \sigma_{\min }\right\}=0
$$

where $\sigma_{\min }$ is some positive number.

Given such a complex measure, we can define a standard non-negative additive set function which is a conventional measure by

$|\lambda|(A)=\sup \left\{\sum_{i=1}^{n}\left|\lambda\left(E_{i}\right)\right|: E_{1}, E_{2}, \ldots, E_{n}\right.$ form a partition of $\left.A\right\}$.

Ash (1972) has shown that this is a legitimate and well defined measure; we need consider those sets for which $(\lambda(A)) \neq 0$. Once $|\lambda|$ has been defined, any equivalent measure (for which sets of zero measure coincide) can be constructed. For some purposes it may be convenient to consider the $|\bmod |^{2}$ measure defined by

$|\lambda|^{2}(A)=\inf \left\{\sum_{i=1}^{n}\left(|\lambda|\left(E_{i}\right)\right)^{2}: E_{1}, E_{2}, \ldots E_{n}\right.$ are disjoint such that $\left.\cup E_{i} \subseteq A\right\}$.

These two measures have the same sets of measure zero.

To clarify the ideas presented so far we offer a few examples.

(i) Binomial distribution.

The random variable is discrete and we have

$\operatorname{Pr}\{\zeta=m\}= \begin{cases}v_{m}=\left(\begin{array}{l}n \\ m\end{array}\right) p_{1}^{m} p_{2}^{n-m}\left(p_{1}+p_{2}\right)^{-n} & \text { if } m=0,1,2, \ldots n \\ 0 & \text { otherwise }\end{cases}$

where $p_{1}$ and $p_{2}$ are any two complex numbers. The normalized mod measure gives rise to the distribution

$$
\operatorname{Pr}\{\zeta=m\}=\mu_{m}=\left(\begin{array}{l}
n \\
m
\end{array}\right)\left|p_{1}\right|^{m}\left|p_{2}\right|^{n-m}\left(\left|p_{1}\right|+\left|p_{2}\right|\right)^{-n}
$$

On the other hand the mod square measure gives rise to the distribution

$$
\operatorname{Pr}\{\zeta=m\}=\lambda_{m}=\left(\begin{array}{l}
n \\
m
\end{array}\right)\left|p_{1}\right|^{2 m}\left|p_{2}\right|^{2 n-2 m}\left(\left|p_{1}\right|^{2}+\left|p_{2}\right|^{2}\right)^{-n} .
$$

It is worth noting that the character of the distribution remains the same in either of the cases, namely, mod and mod square.

(ii) Exponential distribution.

The random variable is continuously distributed with

$$
\mu(\mathrm{d} x)= \begin{cases}\mathrm{e}^{-(\alpha+\mathrm{i} \beta) x}(\alpha+\mathrm{i} \beta) \mathrm{d} x & \text { if } x>0 \quad(\alpha>0) \\ 0 & \text { if } x<0 .\end{cases}
$$

The mod measure gives rise again to an exponential density which, when suitably normalized, is given by

$$
\mu(d x)= \begin{cases}\mathrm{e}^{-\alpha x} \alpha \mathrm{d} x & \text { if } x>0 \\ 0 & \text { if } x<0\end{cases}
$$


(iii) Normal distribution.

In this case the density function is given by

$$
v(\mathrm{~d} x)=\frac{1}{\sqrt{2 \pi \sigma^{2}}} \mathrm{e}^{-(x+\mathrm{i} \alpha)^{2} / 2 \sigma^{2}} \mathrm{~d} x .
$$

The mod measure yields again the normal distribution with zero mean and variance equal to $\sigma^{2}$.

The mod square measure also yields the normal distribution although with a different parameter. We can define the complex Gaussian random variable by

$$
\xi=m+\zeta+\mathrm{i} \eta
$$

where $\zeta$ and $\eta$ are independent random variables and have the same Gaussian distributions with zero mean. This will enable us to define a Gaussian process presently.

(iv) Poisson distribution.

The random variable is discrete and the probability given by

$$
\operatorname{Pr}\{\zeta=n\}= \begin{cases}\mathrm{e}^{-(\alpha+\mathrm{i} \beta)} \frac{(\alpha+\mathrm{i} \beta)^{n}}{n !} & \text { if } n=0,1,2, \ldots \\ 0 & \text { otherwise }\end{cases}
$$

The mod measure yields a Poisson distribution:

$$
\operatorname{Pr}\{\zeta=n\}=\mathrm{e}^{-\rho} \frac{\rho^{n}}{n !}
$$

where $\rho=|\alpha+\mathrm{i} \beta|$. On the other hand, the mod square yields an odd distribution:

$$
\operatorname{Pr}\{\zeta=n\}=\frac{\left(\alpha^{2}+\beta^{2}\right)^{n}}{(n !)^{2}} \frac{1}{I_{0}\left(2 \sqrt{\alpha^{2}+\beta^{2}}\right)} .
$$

We proceed next to define a generalized random sequence (discrete stochastic process) or a generalized random function (arbitrary stochastic process). To do this we note that we need to extend the definition of a generalized random vector $\left(x_{1}, x_{2}, \ldots, x_{n}\right)$. This is done by simply following the procedure recommended by Pitt (1968) (see section 18). We first introduce simple sets as finite unions of rectangular sets of functions $x(t)$ satisfying a finite set of conditions

$$
a_{m} \leqslant x\left(t_{m}\right)<b_{m} .
$$

Since simple sets for a finite set of points $t_{m}$ form a ring, the totality of all such simple sets also form a ring. Then we start with a complex valued additive set function $\mu_{0}(I)$ defined on the ring of simple sets of $\Omega$. If we constrain the values $\mu_{0}(I)$ on the ring of sets associated with $t_{1}, t_{2}, \ldots t_{n}$ be those of a complex probability distribution in $R_{n}$, then it is indeed possible to define a complex measure $\mu(x)$ in $\Omega$ in such a manner that every simple set is measurable and $\mu(I)=\mu_{0}(I)$. This shows that it is possible to deal with an indexed family of complex measures in a consistent way irrespective of whether the index labels a discrete set or a continuum. We call such an indexed family a generalized stochastic process.

The notions of conditional probability measure, Markov property and stationarity can be carried over in toto for such a generalized stochastic process. We shall examine presently the consequences in detail. Now we present some simple examples. 


\subsection{Simple Poisson process}

We recall that the Poisson process can be defined in many different but equivalent ways. We can view a Poisson process as a process of intervals whose lengths form a family of independent and identically distributed (IID) non-negative random variables with a common exponential distribution. For complex measures we introduce $F_{X}(\cdot)$ by

$$
F_{X}(x)=\operatorname{Pr}\{X \leqslant x\}= \begin{cases}1-\mathrm{e}^{-(\alpha+\mathrm{i} \beta) x} & \text { if } x>0 \\ 0 & \text { if } x<0\end{cases}
$$

where $\alpha>0$. This measure generates a mod measure whose normalized density is given by

$$
\alpha \mathrm{e}^{-\alpha x} \mathrm{~d} x
$$

Thus we conclude that the mod measure leads us to a family of ID random variables with a common exponential distribution

$$
F_{X}(x)=1-\mathrm{e}^{-\alpha x} .
$$

Thus the process of IID intervals remains the same under mod measure and hence leads to a Poisson process with $\operatorname{Re}(\alpha+\mathrm{i} \beta)$ as parameter.

A Poisson process can also be viewed as a homogeneous Markov process whose infinitesimal generator is given by

$$
\begin{aligned}
\pi\left(n, n^{\prime}, \Delta\right) & =\operatorname{Pr}\left\{x(t+\Delta)=n \mid x(t)=n^{\prime}\right\} \\
& =\lambda \Delta \delta_{n-1 n^{\prime}}+\delta_{n n^{\prime}}(1-\lambda \Delta)
\end{aligned}
$$

which leads to the result

$$
\begin{aligned}
\pi(n, t) & =\operatorname{Pr}\{X(t)=n \mid X(0)=0\} \\
& =\mathrm{e}^{-\lambda t}(\lambda t)^{n} / n
\end{aligned}
$$

where $\lambda$ is any complex parameter with positive real part. We note that the infinitesimal generator also leads to the set of differential equations

$$
\begin{array}{ll}
\pi^{\prime}(n, t)=-\lambda \pi(n, t)+\lambda \pi(n-1, t) & n>0 \\
\pi^{\prime}(0, t)=-\lambda \pi(0, t) & \\
\pi(n, t)=0 & n<0 .
\end{array}
$$

It is interesting to note that mod measure applied at any stage (either at the level of infinitesimal generator or later) leads to the Poisson process with parameter $|\lambda|$. This is possibly due to the non-diffusive nature of the Poisson process.

\subsection{Yule-Furry Process}

This process can be viewed as a homogeneous Markov process with infinitesimal generator given by

$$
\begin{aligned}
\pi\left(n, n^{\prime}, \Delta\right) & =\operatorname{Pr}\left\{X(t+\Delta)=n \mid X(t)=n^{\prime}\right\} \\
& =(1-\lambda n \Delta) \delta_{n n^{\prime}}+\lambda(n-1) \Delta \delta_{n-1, n^{\prime}}
\end{aligned}
$$

which leads to the result

$$
\begin{aligned}
\pi(n, t) & =\operatorname{Pr}\{X(t)=n \mid X(0)=1\} \\
& =\mathrm{e}^{-\lambda t}\left(1-\mathrm{e}^{-\lambda t}\right)^{n-1} .
\end{aligned}
$$


Now if mod measure is applied at the level of the infinitesimal generator, we are led to the result

$$
\pi(n, t)=\mathrm{e}^{|\lambda| t}\left(1-\mathrm{e}^{-|\lambda| t}\right)^{n-1} .
$$

On the other hand, if mod measure is applied at the end, we have

$$
\pi(n, t)=\left|1-\mathrm{e}^{-\lambda t}\right|^{n-1} /\left(1-\left|1-\mathrm{e}^{-\lambda t}\right|\right) \text {. }
$$

For fixed $t$, the distribution is geometric in either case. The Yule-Furry process can also be viewed as a (non-stationary) point process. Let us fix the time interval and take it as $[0, t]$. Assuming $\operatorname{Pr}\{x(0)=1\}=1$, we can write down the exclusive density (known as the Janossy density in the literature on Poisson processes) as the finite dimensional distribution denoted by

$$
J_{n}\left(t_{1}, t_{2}, \ldots t_{n}\right)
$$

where

$$
J_{n}\left(t_{1}, t_{2} \ldots t_{n}\right) \mathrm{d} t_{1} \mathrm{~d} t_{2} \ldots d, t_{n}
$$

denotes the probability of incidence in each of the intervals $\left(t_{i}, t_{i}+\mathrm{d} t_{i}\right)$ with $0<t_{i}<t, i=1,2, \ldots n$. $J_{n}$ can be computed; for $0<t_{1}<t_{2}<\ldots<t_{n}<t$ we have

$$
\begin{aligned}
J_{n}\left(t_{1}, t_{2} \ldots t_{n}\right) \mathrm{d} t_{1}, \mathrm{~d} t_{2} \ldots \mathrm{d} t_{n} \\
\quad=\mathrm{e}^{-\lambda f_{1}-2 \lambda\left(t_{2}-t_{1}\right)} \ldots-n \lambda\left(t_{n}-t_{n-1}\right) \lambda(2 \lambda) \ldots(n \lambda) \mathrm{d} t_{1} \mathrm{~d} t_{2} \ldots \mathrm{d} t_{n} \mathrm{e}^{-(n+1) \lambda\left(t-t_{n}\right)}
\end{aligned}
$$

so that

$$
J_{n}=n ! \lambda^{n} \mathrm{e}^{-\lambda t} \prod_{i=1}^{n} \mathrm{e}^{-\lambda\left(\varepsilon-t_{i}\right)} .
$$

If mod measure is applied at this stage, then the parameter is obviously $\operatorname{Re} \lambda$ which will lead to results different from either of the alternatives mentioned earlier; this was to be expected.

\section{Markov processes and their identification with standard physical processes}

As suggested earlier, we define a stochastic process as an indexed family of complex measures. The existence of a measure space over which such a stochastic process (or a random function) is defined is proved along lines exactly similar to those adopted by Pitt. Such an indexed family of complex measures is best defined by the conditional structure:

$F_{n}\left(x, t \mid x_{0}, t_{0}, \ldots x_{n}, t_{n}\right)=\operatorname{Pr}\left\{X(t) \leqslant X \mid X\left(t_{0}\right)=x_{0}, X\left(t_{1}\right)=x_{1} \ldots X\left(t_{n}\right)=x_{n}\right\}$

where

$$
t_{0} \leqslant t_{1} \leqslant t_{2} \leqslant \ldots \leqslant t_{n} \leqslant t \quad n=0,1,2, \ldots
$$

or by the density functions

$$
\begin{aligned}
f_{n}\left(x, t ; x_{0}, t_{0} ; x_{1}, t_{1}, \ldots x_{n}, t_{n}\right) \mathrm{d} x & \\
& =\operatorname{Pr}\left\{x<X(t) \leqslant x+\mathrm{d} x \mid X\left(t_{0}\right)=x_{0}, X\left(t_{1}\right)=x_{1}, \ldots X\left(t_{n}\right)=x_{n}\right\} .
\end{aligned}
$$

A process is called Markov if

$$
F_{n}\left(x, t \mid x_{0}, t_{0}, x_{1}, t_{1}, \ldots x_{n}, t_{n}\right)=F_{2}\left(x, t \mid x_{n}, t_{n}\right)
$$


or

$$
f_{n}\left(x, t \mid \eta_{0}, t_{0}, x_{1}, t_{1} \ldots x_{n}, t_{n}\right)=f_{2}\left(x, t \mid x_{n}, t_{n}\right)
$$

for every choice of $x_{0}, x_{0}, x_{1}, x_{2} \ldots x_{n}$ and $t_{0}, t_{1}, t_{2} \ldots t_{n}$ subject to $t_{0} \leqslant t_{1} \leqslant t_{2} \ldots \leqslant t_{n} \leqslant t$. A similar definition holds when the process is discrete-valued or $t$ is discrete-valued or both. If we denote by $F(x, t)$ the probability

$$
\operatorname{Pr}\{X(t) \leqslant x\} \text { or } f(x, t) \mathrm{d} x=\operatorname{Pr}\{x \leqslant x(t) \leqslant x+\mathrm{d} x\}
$$

then we have the Chapman-Kolmogorov relation

$$
f_{2}\left(x, t \mid x_{0}, t_{0}\right)=\int f_{2}\left(x, t \mid x^{\prime}, t^{\prime}\right) f_{2}\left(x^{\prime}, t^{\prime} \mid x_{0}, t_{0}\right) d x^{\prime} \quad \text { for } \forall t^{\prime} \in\left(t_{0}, t\right)
$$

and

$$
f_{2}(x, t)=\int f_{2}\left(x, t \mid x^{\prime}, t^{\prime}\right) f\left(x^{\prime}, t^{\prime}\right) \mathrm{d} x^{\prime} \quad \text { for } \forall t^{\prime}<t
$$

(provided $t^{\prime}$ is in the domain of definition of the process.) We may write this in a more suggestive fashion as

$$
\psi\left(x_{2}, t_{2}\right)=\int K\left(x_{2}, t_{2} \mid x_{1}, t_{1}\right) \psi\left(x_{1}, t_{1}\right) d x_{1} .
$$

(i) We now make a special choice leading to the Schrödinger equation. Take $t_{2}=t_{1}+\epsilon(\epsilon$ small). We choose

$$
\begin{aligned}
-K & =\frac{1}{A} \exp \left\{\frac{\mathrm{i}}{\hbar} m \frac{\left(x_{2}-x_{1}\right)^{2}}{\epsilon}\right\} \exp \left\{-\frac{\mathrm{i}}{\hbar} \epsilon V\left(\frac{x_{1}+x_{2}}{2}, t\right)\right\} \\
A & =\left(\frac{2 \pi \mathrm{i} \hbar \epsilon}{m}\right)^{1 / 2} .
\end{aligned}
$$

For such a choice we have for arbitrarily small $\epsilon$

$$
\int K \mathrm{~d} x_{2}=1 \text {. }
$$

(This proves that $K$ is a genuine complex probability measure.)

Then we have

$$
\mathrm{i} \hbar \frac{\partial \psi}{\partial t}=-\frac{\hbar^{2}}{2 m} \frac{\partial^{2} \psi}{\partial x^{2}}+V(x, t) \psi
$$

$K_{2}$ also satisfies an identical equation if we apply this to equation (3.7).

This is in fact a special case of the general Fokker-Planck equation.

(ii) Fokker-Planck equation. We note that $f_{2}$ satisfies the Chapman-Kolmogorov equation, choose $t_{0}=0$ and assume time homogeneity:

$$
f_{2}\left(x \mid x_{0}, t+\Delta\right)=\int f_{2}(x \mid z, \Delta) f_{2}\left(z \mid x_{0}, t\right) \mathrm{d} z .
$$

Now we put some constraints:

$$
a_{n}(z, \Delta)=\int(x-z)^{n} f_{2}(x \mid z \Delta) d a
$$

and that $a_{n} / \Delta$ has a non-zero limit for $n=1$ and 2:

$$
A(z)=\lim _{\Delta \rightarrow 0} \frac{a_{1}(z, \Delta)}{\Delta}=\quad B(z)=\lim _{\Delta \rightarrow 0} \frac{a_{2}(z, \Delta)}{\Delta} .
$$


To obtain an equation for $f_{2}$, we consider

$$
\int \frac{\partial f_{2}\left(x \mid x_{0}, t\right)}{\partial t} \phi(x) d x
$$

where $\phi$ is a smooth function that tends to zero sufficiently fast as $x \rightarrow \pm \infty$ :

$$
\int \frac{\partial f_{2}}{\partial t} \phi(x) \mathrm{d} x=\lim _{\Delta \rightarrow 0} \int \phi(x) \frac{\left[f_{2}\left(x \mid x_{0}, t+\Delta\right)-f_{2}\left(x \mid x_{0}, t\right]\right.}{\Delta} \mathrm{d} x .
$$

Now use the Chapman-Kolmogorov relation:

$$
\int \frac{\partial f_{2}}{\partial t} \phi(x) \mathrm{d} x=\lim _{\Delta \rightarrow 0} \frac{1}{\Delta}\left[\int \mathrm{d} x \phi(x) \int f_{2}(x \mid z, \Delta) f_{2}\left(z \mid x_{0}, t\right) \mathrm{d} z-\int d_{2} \phi(x) f_{2}\left(x \mid x_{0}, t\right)\right] \text {. }
$$

In the first term we expand $\phi(x)$ in powers of $x-z$ to obtain

$$
\begin{aligned}
\int \frac{\partial f_{2}}{\partial t} \phi(x) \mathrm{d} x & \\
= & \frac{1}{\Delta}\left[\iint \phi(z) \mathrm{d} z f_{2}(x \mid z, A) f_{2}\left(z \mid X_{0}, t\right) \mathrm{d} X-\int \mathrm{d} x \phi(x) f_{2}\left(x \mid x_{0}, t\right)\right] \\
& +\int\left[A(z) \phi^{\prime}(z)+\frac{1}{2} B(z) \phi^{\prime \prime}(z)\right] f_{2}\left(z \mid x_{0}, t\right) \mathrm{d} z \\
= & \int\left[A(B) \phi^{\prime}(z)+\frac{1}{2} B(z) \phi^{\prime \prime}(B)\right] f_{2}\left(z \mid x_{0}, t\right) \mathrm{d} z .
\end{aligned}
$$

Integrating by by parts, we obtain

$$
\int \frac{\partial f_{2}}{\partial t} \phi(x) \mathrm{d} x=\int\left\{\frac { \partial } { \partial z } \left[A(z) f_{2}\left(z \mid x_{0}, t\right]-\frac{1}{2} \frac{\partial^{2}}{\partial z^{2}}\left[B(z) f_{2}\left(z \mid x_{0}, t\right]\right\} \phi(z) \mathrm{d} z .\right.\right.
$$

Since this holds for $\forall \phi$, we have the equation

$$
\frac{\partial f_{2}\left(x \mid x_{0}, t\right)}{\partial t}=-\frac{\partial}{\partial x}\left[f_{2}\left(x \mid x_{0}, t\right) A(x)\right]+\frac{1}{2} \frac{\partial^{2}}{\partial x^{2}}\left[f_{2}\left(x \mid x_{0}, t\right) B(x)\right] .
$$

Thus the kernel function can'satisfy a very general type of equation, the Schrödinger equation being a very special case. We have considered a homogenous process; we can relax the same, in which case we are led to time-dependent potentials.

Let us consider a special case when

$$
B(x)=\text { a constant }=-\frac{\mathrm{i} \hbar}{m} \quad \text { (say). }
$$

Then we can use the standard transformation

$$
f_{2}\left(x \mid x_{0} t\right) \exp \left(-\frac{1}{2} \int_{0}^{x} A(\zeta) d \zeta\right)=g_{2}\left(x \mid x_{0}, t\right)
$$

in which case the equation reduces to

$$
\mathrm{i} \hbar \frac{\partial g_{2}\left(x \mid x_{0} t\right)}{\partial t}=-\frac{\hbar^{2}}{2 m} \frac{\partial^{2} g_{2}\left(x \mid x_{0}, t\right)}{\partial x^{2}}+g_{2}\left(x \mid x_{0}, t\right)\left[\frac{m}{2}\{A(x)\}^{2}-\frac{\mathrm{i} \hbar \mathrm{d} A(x)}{\mathrm{d} x}\right] \text {. }
$$

This is the Schrödinger equation!

If $A$ were to be a function of $t$ also, we would have an additional term

$$
-\frac{\mathrm{i} \hbar}{2} \int_{0}^{2} \frac{\partial A(\zeta, t)}{\partial t} \mathrm{~d} \zeta
$$

within the square bracket on the right-hand side. 


\section{Jump processes}

In this case we can define a Markov process and then specify the same by the infinitesimal generator:

$$
\begin{aligned}
\operatorname{Pr}\{X(t+\Delta) & =n \mid X(t)=m\} \\
& =\delta_{n m}\{1-\Delta[v+\alpha+m \lambda+m \mu]\}+\delta_{n m+1}[v \Delta+ \\
+\delta_{n m-1}[\alpha \Delta+m \mu \Delta]+O(\Delta) &
\end{aligned}
$$

where $\lambda, \mu, v, \alpha$ are complex constants. Several standard processes can be subsumed under this general process:

(i) A pure birth process of the Yule type (popularly known as Furry process although Furry rediscovered the Yule process 21 years after Yule!) $\nu=\mu=\alpha=0$

(ii) A birth and death process: $v=\alpha=0$.

(iii) Poisson process: $\lambda=v=\alpha=0$.

(iv) A population process of thermal photons obeying BE statistics: $\alpha=0, \lambda=\nu$.

(v) A population process of coherent photons: same as (iii) above.

(vi) A single channel queuing process with Poisson arrivals: $\lambda=-\mu=0$ (with same appropriate boundary conditions at $n=0$ ).

(vii) A random telegraph signal process: take the state space modulo $\{0,1\}$. Set $\lambda=\mu=0$.

For purposes of illustration let us consider the case of population process of photons by setting $\alpha=0$. Introducing:

$$
\begin{aligned}
\pi(n, t) & =\operatorname{Pr}\{X(t)=n \mid X(0)=0\} \\
G(u, t) & =\sum \pi(n, t) u^{n} \quad|u|<1 \\
& =E\left[u^{X(t)}\right] \\
g(u, t) & =E\left[u^{X(t)} \mid X(0)=1, v=0\right]
\end{aligned}
$$

we obtain (by interpreting the process as a branching process)

$$
\begin{aligned}
& \frac{\partial g(u, t)}{\partial t}=-(\lambda+\mu) g(u, t)+\lambda[g(u, t)]^{2}+\mu \\
& \frac{\partial G(u, t)}{\partial t}=-v G(u, t)+v G(u, t) g(u, t) \\
& g(u, 0)=u, \quad G(u, 0)=1 .
\end{aligned}
$$

Solving we obtain (for $v=\lambda$ )

$$
G(u, t)=\frac{(\mu-\lambda)}{\left(\mu-\lambda z+\lambda(z-1) \mathrm{e}^{-(\mu-\lambda) t}\right)} .
$$

If we set $|\mu|>|\lambda|$, then we have a limit distribution:

$$
\begin{aligned}
& \lim _{E \rightarrow \infty} G(u, t)=\frac{\mu-\lambda}{\mu-\lambda z}=\text { (BE distribution) } \\
& p_{E}(n)=\frac{\mu-\lambda}{\mu}\left(\frac{\lambda}{\mu}\right)^{n} \quad \text { (equilibrium distribution). }
\end{aligned}
$$

If we use mod measure,

$$
\left.p_{E}(n)\right|_{\bmod \mu} \rightarrow\left(1-\left|\frac{\lambda}{\mu}\right|\right)\left|\frac{\lambda}{\mu}\right|^{n} .
$$


If we use mod square measure

$$
\left.p_{E}(n)\right|_{(\bmod \mu)^{2}} \rightarrow\left(1-\left|\frac{\lambda}{\mu}\right|^{2}\right)\left|\frac{\lambda}{\mu}\right|^{2 n} .
$$

It is interesting to note that either measure retains the geometric (BE) nature of the distribution. The population point process models of photon population have been studied (Jakeman 1980, Shepherd 1981, Jakeman and Shepherd 1987) using positive definite rate constants. An extension of these models to include non-Markov evolution had been provided by Srinivasan $(1988,1990)$ and Srinivasan and Sridharan (1990). The above model with complex rates may be made appropriate to describe photon population.

As the next example, consider the telegraph signal process. This is just a two-valued process. The different probabilities can be explicitly evaluated using the Markov nature of the process:

$$
\begin{aligned}
& \pi_{00}(t)=\operatorname{Pr}[X(t)=0 \mid X(0)=1]=\frac{\alpha}{\alpha+v}+\frac{v}{\alpha+v} \mathrm{e}^{-(\lambda+v) t} \\
& \pi_{11}(t)=\operatorname{Pr}[X(t)=1 \mid X(0)=1]=\frac{v}{\alpha+v}+\frac{\alpha}{\alpha+v} \mathrm{e}^{-(\alpha+v) t} \\
& \pi_{00}(\infty)=\frac{\alpha}{\alpha+v}, \pi_{11}(\infty)=\frac{v}{\alpha+v} \\
& \left.\pi_{00}(\infty)\right|_{\bmod \mu} \rightarrow\left|\frac{\alpha}{\alpha+v}\right|,\left.\pi_{11}(\infty)\right|_{\bmod \mu} \rightarrow\left|\frac{v}{\alpha+v}\right|
\end{aligned}
$$

On normalization

$$
\begin{aligned}
& \pi_{00}(\infty)=\frac{\left|\frac{\alpha}{\alpha+\nu}\right|}{\left(\left|\frac{\alpha}{\alpha+\nu}\right|+\left|\frac{\nu}{\alpha+\nu}\right|\right)}=\frac{|\alpha|}{|\alpha|+|\nu|} \\
& \pi_{11}(\infty)=\frac{\left|\frac{v}{\alpha+\nu}\right|}{\left(\left|\frac{\alpha}{\alpha+\nu}\right|+\left|\frac{\nu}{\alpha+\nu}\right|\right)}=\frac{|\nu|}{|\alpha|+|\nu|} \\
& \left.\pi_{01}(\infty)\right|_{\bmod \mu^{2}}=\frac{|\alpha|^{2}}{|\alpha|^{2}+|\nu|^{2}} \pi_{11}(\infty)_{\bmod \mu^{2}}=\frac{|\nu|^{2}}{|\alpha|^{2}+|\nu|^{2}}
\end{aligned}
$$

Instead of the complex rates, we need the mod or (mod) ${ }^{2}$ of the rates. The above process has been discussed in detail by one of us (Srinivasan 1988) in connection with point process models of cavity radiation and detection. Erber et al (1989) have discussed the process in connection with the modelling of dark and bright periods generated by resonance fluorescence and quantum jumps. They have of course used positive-definite probabilities; it may be worthwhile to study these models using complex probabilities. A very special model of the telegraph signal process was considered by Ramakrishnan et al (1960) as well as Gaveau et al (1984). While the former were concerned with neutron transport and backscattering, the latter used the model to obtain one-dimensional relativistic electron motion. Srinivasan (1963) had dealt with the same process in connection with a model of emulsion polymerization. We shall presently show how a model of this type within the framework of complex measures can lead to the Dirac equation of an electron with a relativistic potential.

Now we proceed to discuss conditional complex probability amplitudes and their properties. Such amplitudes have been studied right from the time Schrödinger introduced his wave equation. It was Dirac (1933) who proposed the action principle to formulate the basic problem of quantum mechanics in terms of the composition law for the bracket $\left(q_{t} \mid q_{\tau}\right)$ which in turn had been extensively used by Feynman (1948) for the calculation of 
probability amplitudes in specific problems. We will now demonstrate that the composition law of Dirac comes out naturally as a consequence of the Markov property of generalized stochastic process (of complex measures) and that 'the sum over paths' is the realization of the addition of the complex measures of inclusive events.

\section{Conditional expectations: the propagating amplitude}

- Let us now define conditional amplitudes.

$f_{i k k_{1} k_{2} \cdots}\left(x \mid y ; y_{1}, y_{2}, \cdots\right) \lim _{\Delta \rightarrow 0} \operatorname{Pr}\left\{x \leqslant x_{j} \leqslant x+\Delta \mid x_{k}=y ; x_{k_{l}}=y_{1}, \cdots\right\} / \Delta$.

This conditional probability has the Markov property if

$$
f_{j k k_{1} k_{2} \ldots}\left(x \mid y ; y_{1}, \cdots\right)=f_{j k}(x \mid y) \quad k>k_{1} \neq k_{2} \neq \cdots .
$$

To define the Markov property it is not necessary to restrict attention to real conditional probabilities: it could equally well be applied to complex conditional probability amplitudes.

We recognize that $f_{j k}(x \mid y)$ is a complex probability amplitude with respect to $x$ indexed by the prior value $y$ and the labels $j, k, j>k$. This amplitude satisfies the ChapmanKolmogorov relation

$$
f_{j k}(x \mid y)=\int f_{j \ell}(x \mid z) f_{\ell k}(z \mid y) \mathrm{d} z \quad j<\ell<k .
$$

Thus the complex-indexed amplitudes have the Markov chain structure. The indices $j, k, \ell$ are ordered parameters associated with the conditional distributions and may be discrete.

However, if $j, k, \ell$ are treated as (continuous) time labels, one could consider infinitesimal intervals and differential equations of motion. They would then 'propagate' the complex amplitudes. The conditional amplitudes $f(x \mid y)$ then become the unfolding of the law of time evolution: the Hamiltonian

$$
\mathrm{i} \frac{\partial}{\partial t} \leftrightarrow H\left(x, \frac{\partial}{\partial x}, t\right)
$$

leads to the time-ordered exponential:

$$
f_{t_{1} t_{2}}(x \mid y)=T\left(\exp \left(\mathrm{i} \int_{t_{t}}^{t_{2}} H(t) \mathrm{d} t\right)\right)(x \mid y) .
$$

Since the operators at different times may not commute, the solution for the time-ordered exponential is the Green function

$$
G_{t_{1} t_{2}}(x \mid y)= \begin{cases}\sum_{\alpha} \varphi_{\alpha}(x, t) \varphi_{\alpha}^{*}\left(y, t_{2}\right) & t_{2} \geqslant t_{1} \\ 0 & t_{2} \geqslant t_{1}\end{cases}
$$

where $\left\{\varphi_{\alpha}(x, t)\right\}$ are a complete set of solutions to the homogeneous equations

$$
\left\{\mathrm{i} \frac{\partial}{\partial t}-H\left(x, \frac{\partial}{\partial x}, t\right)\right\} \varphi(x, t)=0 .
$$

The choice of the retarded function is in accordance with the directed property of the Chapman-Kolmogoroy equation.

In dealing with the Hamiltonian in this form, it should contain the displacement operator with respect to $x$ either explicitly or implicitly. Hence the complete set of operators $x, \partial / \partial x$ are non-commuting; in quantum mechanics, or more generally in wave theories, this is clearly seen. In the former case we have the momentum operator $p$ which is realised as 
$(\hbar / \mathrm{i})(\partial / \partial x)$. In classical particle mechanics, the Hamiltonian operator is in fact the Poisson bracket of the Hamilton function

$$
H=\frac{\mathrm{i}}{\hbar}[h(q, p), \cdot]_{\mathrm{Pb}} \cdot
$$

The Green function $G(x, y)$ is, in all cases, a function of $x, y$ indexed by $t$.

While the forward propagation can be carried out for arbitrary future times, the tracing back, in general, can be done only for finite times. In general, the Markov processes are contractive; cyclic processes are exceptions. But with complex amplitudes we can have self-adjoint Hamiltonians with the probability of either forward or backward propagation. Of course, we could also have contractive semigroups of evolutions. The two classes of processes are distinguished in terms of the spectrum of the evolution operator. For the symmetric case the spectrum is unimodular while in the unsymmetric case there are spectra within the unit circle in the complex plane.

\section{Huyghens' principle}

The generic physical example of conditional expectation is the (generalized) Huyghens' principle for time dependent (optical) pulses. In this case (for scalar -light) the conditional probability $f(x \mid y)$ is the response at $y$ for unit stimulus at $x$ at an earlier time. The Huyghens' kernel satisfies both the Green function equation

$$
\left(\mathrm{i} \frac{\partial}{\partial t}-\sqrt{-\Delta^{2}}\right) f_{0 t}(x, y)=\delta(x-y) \delta(t)
$$

and the Markov property

$$
f_{t_{1} f_{2}}(x \mid y)=\int \mathrm{d} z f_{t_{1}}(x \mid z) f_{t t_{2}}(z \mid y) ; t_{1}>t>t_{2} .
$$

If the initial condition is given in terms of $\varphi_{t_{0}}(x)$ then

$$
\varphi_{t}(x)=\int \mathrm{d} y f_{t_{0}}(x \mid y) \varphi_{t_{0}}(y)
$$

Mutatis mutandis, this formalism applies equally well to other wave-like structures including acoustics and quantum mechanics.

If one is interested in only first-order (in time) differential equations, the retarded solution is the natural solution. When there is a second-order equation there are other possibilities. If the propagation is in a homogenous medium, one can distinguish invariantly between positive- and negative-frequency solutions and restrict attention to the positive-frequency solutions only. These are the analytic signals of Gabor (1946) and Wolf (1955); see also Sudarshan (1969). In a way, we realize this even for the Schrödinger equation written as a two-component real equation.

But when external fields (or self-interactions) are included, there are transitions between the positive- and negative-energy states and so we cannot restrict attention to positive energies only. We can still deal with retarded functions but the causal Stuckelberg-Feynman time-ordered Green function, which propagate positive frequencies forward and negative frequencies backward, is more useful:

$$
F_{o t}(x \mid y)=\left\{\begin{array}{lll}
\varphi_{\alpha}(x, t) \varphi_{\alpha}^{*}(y, 0) & E_{\alpha}>0 & t<0 \\
\varphi_{\beta}(x, t) \varphi_{\beta}^{*}(y, 0) & E_{\beta}<0 & t>0
\end{array}\right.
$$




\section{Derivation of the Schrödinger and Dirac equations}

Define the complex measure $\pi(y, t)$ and the conditional probability $p(x, z, t, \Delta) \mathrm{d} x$ by

$$
\begin{aligned}
& p(x, z ; t ; \Delta) \mathrm{d} x=\operatorname{Pr}\{x<y(t=\Delta)<x+\mathrm{d} x \mid y(t)=z\} \\
& \pi(y, t) \mathrm{d} y=\operatorname{Pr}\{y<y(t)<y+\mathrm{d} y\}=\int \mathrm{d} z p(y, z, t, 0) .
\end{aligned}
$$

The Chapman-Kolmogorov relations obeyed are

$$
\pi(y, t+\Delta)=\int \pi(z, t) p(y, z, t, \Delta) \mathrm{d} z .
$$

Define

$$
A_{n}(z, t, \Delta)=\int(y-z)^{n} p(y, z, t, \Delta) \mathrm{d} y .
$$

Then for any smooth $\psi(y)$ with the expansion

$$
\psi(y)=\sum_{0}^{\infty}(y-z)^{n} \psi^{(n)}(z) / n !
$$

there is the relation

$$
\begin{aligned}
\int \pi(y, t+\Delta) & \psi(y) \mathrm{d} y=\sum_{0}^{\infty} \int \pi(z, t) \psi^{(n)}(z) A_{n}(z, t, \Delta) \mathrm{d} z \\
& =\sum_{0}^{\infty} \int\left\{\left(\frac{-\partial}{\partial z}\right)^{n}\left[\pi(z, t) A_{n}(z, t, \Delta)\right]\right\} \psi(z) \mathrm{d} z .
\end{aligned}
$$

If we now require the following conditions suggested by a generalized random walk:

$$
\begin{aligned}
& \lim _{\Delta \rightarrow 0} \frac{A_{1}(z, t, \Delta)}{\Delta}=A(z, t) \\
& \lim _{\Delta \rightarrow 0} \frac{A_{2}(z, t, \Delta)}{\Delta}=B(z, t) \\
& \lim _{\Delta \rightarrow 0} \frac{A_{n}(z, t, \Delta)}{\Delta}=0(\Delta) \quad n \geqslant 3 .
\end{aligned}
$$

The following relations hold for arbitrary smooth $\psi(y)$ :

$\frac{\partial \pi(y, t)}{\partial t}=-a(y, t) \pi(y, t)-c(y, t) \frac{\partial \pi(y, t)}{\partial y}+\frac{1}{2} b(y, t) \frac{\partial^{2} \pi(y, t)}{\partial y^{2}}$

where $a, b, c$ are related to $A, B, C$ by simple relations. If we compare it with the Schrödinger equation

$$
\mathrm{i} \hbar \frac{\partial}{\partial t} \psi=-\frac{\hbar^{2}}{2 m} \nabla^{2} \psi
$$

we get the correspondence

$$
\begin{aligned}
& \frac{\hbar^{2}}{2} b(y, t)=-\frac{\hbar^{2}}{2 m} \\
& c(y, t)=a(y, t)=0 .
\end{aligned}
$$

On the other hand, the Schrödinger equation with an external scalar potential $\phi(x)$ and vector potential $A(z)$ given by

$$
\mathrm{i} \hbar \frac{\partial}{\partial t} \psi=\left\{-\frac{\hbar^{2}}{2 m}(\nabla-\mathrm{i} e A)^{2}+\phi\right\} \psi
$$


$(h / \mathrm{i})(\partial / \partial x)$. In classical particle mechanics, the Hamiltonian operator is in fact the Poisson bracket of the Hamilton function

$$
H=\frac{\mathrm{i}}{\hbar}[h(q, p), \cdot]_{\mathrm{Pb}} \text {. }
$$

The Green function $G(x ; y)$ is, in all cases, a function of $x, y$ indexed by $t$.

While the forward propagation can be carried out for arbitrary future times, the tracing back, in general, can be done only for finite times. In general, the Markov processes are contractive; cyclic processes are exceptions. But with complex amplitudes we can have self-adjoint Hamiltonians with the probability of either forward or backward propagation. Of course, we could also have contractive semigroups of evolutions. The two classes of processes are distinguished in terms of the spectrum of the evolution operator. For the symmetric case the spectrum is unimodular while in the unsymmetric case there are spectra within the unit circle in the complex plane.

\section{Huyghens' principle}

The generic physical example of conditional expectation is the (generalized) Huyghens' principle for time dependent (optical) pulses. In this case (for scalar light) the conditional probability $f(x \mid y)$ is the response at $y$ for unit stimulus at $x$ at an earlier time. The Huyghens' kernel satisfies both the Green function equation

$$
\left(\mathrm{i} \frac{\partial}{\partial t}-\sqrt{-\Delta^{2}}\right) f_{0 t}(x, y)=\delta(x-y) \delta(t)
$$

and the Markov property

$$
f_{t_{1} f_{2}}(x \mid y)=\int \mathrm{d} z f_{t_{1} t}(x \mid z) f_{t_{2}}(z \mid y) ; t_{1}>t>t_{2} .
$$

If the initial condition is given in terms of $\varphi_{t_{0}}(x)$ then

$$
\varphi_{t}(x)=\int \mathrm{d} y f_{t t_{0}}(x \mid y) \varphi_{t_{0}}(y) .
$$

Mutatis mutandis, this formalism applies equally well to other wave-like structures including acoustics and quantum mechanics.

If one is interested in only first-order (in time) differential equations, the retarded solution is the natural solution. When there is a second-order equation there are other possibilities. If the propagation is in a homogenous medium, one can distinguish invariantly between positive- and negative-frequency solutions and restrict attention to the positive-frequency solutions only. These are the analytic signals of Gabor (1946) and Wolf (1955); see also Sudarshan (1969). In a way, we realize this even for the Schrödinger equation written as a two-component real equation.

But when external fields (or self-interactions) are included, there are transitions between the positive- and negative-energy states and so we cannot restrict attention to positive energies only. We can still deal with retarded functions but the causal Stuckelberg-Feynman time-ordered Green function, which propagate positive frequencies forward and negative frequencies backward, is more useful:

$$
F_{o t}(x \mid y)=\left\{\begin{array}{lll}
\varphi_{\alpha}(x, t) \varphi_{\alpha}^{*}(y, 0) & E_{\alpha}>0 & t<0 \\
\varphi_{\beta}(x, t) \varphi_{\beta}^{*}(y, 0) & E_{\beta}<0 & t>0
\end{array}\right.
$$


The difference $f(x \mid y)-F(x \mid y)$ is a solution of the homogenous equation

$$
f(x \mid y)-F(x \mid y)=\sum_{E_{\beta}<0} \varphi_{\beta}(x, t) \varphi_{\beta}(y, 0) .
$$

The Huyghens' amplitude and the Green function are time dependent functions. For a single step transition one can take the time Fourier transformation to get the monochromatic, time independent Green function

$$
f^{(E)}(x \mid y)=\sum_{\alpha} \frac{\varphi_{\alpha}(x) \varphi_{\alpha}^{*}(y)}{E-E_{\alpha}} .
$$

This amplitude does not satisfy the Markov property; and is not in accordance with Huyghens' principle.

\section{Random diffusion with a complex measure}

Define random variables $x_{k}$ (independent and identically distributed) with

$$
\begin{aligned}
& \operatorname{Pr}\left\{x_{k}=+1\right\}=p+\mathrm{i} q \\
& \operatorname{Pr}\left\{x_{k}=-1\right\}=\bar{p}+\mathrm{i} q \\
& \operatorname{Pr}\left\{x_{k}=+0\right\}=-2 \mathrm{i} q .
\end{aligned}
$$

The special choice leads to real $E\left[x_{k}\right]$ :

$$
E\left[x_{k}\right]=p-\bar{p}
$$

and

$$
\text { Prob }\left\{x_{k}=+1,0,-1\right\}=1 \text {. }
$$

Define the stochastic process $\left\{Y_{n}: n=0,1,2, \cdots\right\}$ by

$$
Y_{n}=\sum_{1}^{n} x_{k} \quad \operatorname{Pr}\left\{Y_{0}=0\right\}=1 .
$$

Then $\left\{Y_{n}\right\}$ is a random walk process and $\operatorname{Pr}\left\{Y_{n}=j\right\}$ can be computed using multinomial coefficients. Note

$$
\begin{aligned}
& E\left[Y_{n}\right]=n(p-\bar{p}) \\
& \operatorname{Var}\left[Y_{n}\right]=2 n(2 p \bar{p}+\mathrm{i} q) .
\end{aligned}
$$

Now we pass on to a continuous version by setting $t=n \Delta$ (the steps being at intervals of length $\Delta)$ and the magnitude of the step $\delta y$ so that $\left(Y_{n} \mapsto Y_{t}\right)$

$$
\begin{aligned}
& E\left[Y_{t}^{*}\right]=t(p-\bar{p}) \frac{\delta y}{\Delta} \\
& \operatorname{Var} Y_{t}^{*}=2(2 p \bar{p}+\mathrm{i} q) t \frac{(\delta y)^{2}}{\Delta} .
\end{aligned}
$$

Note that the initial distribution is concentrated at the origin if we use the continuous density $\pi(y, t), \pi(y, 0)=\delta(y)$.

We define

$$
\tilde{Y}_{t}=Y_{t}^{*}-E\left[Y_{t}^{*}\right]=Y_{t}^{\alpha}-t(p-\bar{p}) \frac{\delta y}{\Delta} .
$$


Note that $\tilde{Y}_{t}$ is also initially concentrated at the origin. We note the process $Y_{t}^{*}$ and hence $\tilde{Y}_{t}$ are Markovian and hence satisfy the Chapman-Kolmogorov equation. By expressing $\pi(y, t)$ in terms of $\pi(y, t-\delta t)$, we obtain

$$
\begin{aligned}
\pi(y, t)=\pi(y+(p-\bar{p}) \delta y-\delta y, t-\Delta)(p+\mathrm{i} q)+\pi(y+(p-\bar{p}) \delta y+\delta y, t-\Delta) \bar{p}+\mathrm{i} q) \\
+\pi(y+(p-\bar{p}) \delta y, t-\Delta)(-2 \mathrm{i} q) .
\end{aligned}
$$

The above equation follows from the discrete version

$\pi(s, n)=\pi(s-1, n-1)(p+\mathrm{i} q)+\pi(s+1, n-1)(\bar{p}+\mathrm{i} q)+\pi(s, n-1)(-2 \mathrm{i} q)$.

Notice that in order that the particle be in position $y$ at $t$, at $t-\Delta$ it should have been at $y+(p-\bar{p}) \delta y$, where the second term is the amount by which it slides back since we have subtracted the expected value; and the third term is the positive jump. Now expand the right-hand side with respect to $y$ :

$$
\begin{gathered}
\pi(y, t)=\pi(y, t-\Delta)+\frac{\partial \pi}{\partial y}\{\delta y(p-\bar{p}-[p+\bar{p}])(p+\mathrm{i} q) \\
+(p-\bar{p}+[p+\bar{p}]) \delta y(\bar{p}+\mathrm{i} q)+(p-\bar{p})(-2 \mathrm{i} q)\} \\
\quad+\frac{1}{2} \frac{\partial^{2} \pi}{\partial y^{2}}\left\{(-2 \bar{p}) \delta y(\bar{p}+\mathrm{i} q)+(2 p)^{2}(\delta y)^{2}(\bar{p}+\mathrm{i} q)+(p-\bar{p})^{2}(\delta y)^{2}(-2 \mathrm{i} q)\right\} \\
\frac{\partial \pi(y, t)}{\partial t}=\frac{\sigma^{2}}{2}(4 p \bar{p}+2 \mathrm{i} q) \frac{\partial^{2} \pi}{\partial y^{2}} \quad \text { where } \quad \sigma^{2}=\lim \frac{(\Delta y)^{2}}{\Delta t} .
\end{gathered}
$$

The equation with the initial condition $\pi(y, 0)=\delta(y)$ yields, for $t>0$, the broadening Gaussian

$$
\pi(y, t)=\left\{2 \pi \sigma^{2} t(4 p \vec{p}+2 \mathrm{i} q)\right\}^{-1 / 2} \exp \left\{\frac{-y^{2}}{2 \sigma^{2} t(4 p \bar{p}+2 \mathrm{i} q)}\right\} .
$$

Note that, as expected, the diffusion is proportional to $t$.

$$
\begin{aligned}
& E\left[\tilde{y}_{t}\right]=0 \\
& \operatorname{Var}\left[\tilde{y}_{t}\right]=(4 p \bar{p}+2 \mathrm{i} q) \sigma^{2} t .
\end{aligned}
$$

In the above analysis we prove a limit theorem that in the random walk/diffusion limit $y_{n} \rightarrow \tilde{y}_{t}$ in probability and that the distribution is normal.

In these calculations, if we had used either the (mod) measure or the (mod) ${ }^{2}$ measure the resulting solutions would have been normal and diffusive with suitably changed parameters.

The case of $p \bar{p}=0$, that is, $p=0,1$, is esspecially interesting in that we can obtain the Schrödinger equation in the absence of potentials:

$$
\mathrm{i} \frac{\partial}{\partial t} \pi(y, t)=-\sigma^{2} q \frac{\partial^{2} \pi}{\partial y^{2}}
$$

where

$$
\left(\frac{2 \sigma^{2} q}{\hbar}\right)^{-1}=m
$$

is the free particle mass in the Schrödinger equation

$$
\mathrm{i} \hbar \frac{\partial}{\partial t} \psi=-\frac{\Delta^{2} \hbar^{2}}{2 m} \psi
$$

With suitably enlarged starting point we can also derive Dirac's relativistic equation. 


\section{Derivation of the Schrödinger and Dirac equations}

Define the complex measure $\pi(y, t)$ and the conditional probability $p(x, z, t, \Delta) \mathrm{d} x$ by

$$
\begin{aligned}
& p(x, z ; t ; \Delta) \mathrm{d} x=\operatorname{Pr}\{x<y(t=\Delta)<x+\mathrm{d} x \mid y(t)=z\} \\
& \pi(y, t) \mathrm{d} y=\operatorname{Pr}\{y<y(t)<y+\mathrm{d} y\}=\int \mathrm{d} z p(y, z, t, 0) .
\end{aligned}
$$

The Chapman-Kolmogorov relations obeyed are

$$
\pi(y, t+\Delta)=\int \pi(z, t) p(y, z, t, \Delta) \mathrm{d} z .
$$

Define

$$
A_{n}(z, t, \Delta)=\int(y-z)^{n} p(y, z, t, \Delta) \mathrm{d} y .
$$

Then for any smooth $\psi(y)$ with the expansion

$$
\psi(y)=\sum_{0}^{\infty}(y-z)^{n} \dot{\psi}^{(n)}(z) / n !
$$

there is the relation

$$
\begin{aligned}
\int \pi(y, t+\Delta) & \psi(y) \mathrm{d} y=\sum_{0}^{\infty} \int \pi(z, t) \psi^{(n)}(z) A_{n}(z, t, \Delta) \mathrm{d} z \\
= & \sum_{0}^{\infty} \int\left\{\left(\frac{-\partial}{\partial z}\right)^{n}\left[\pi(z, t) A_{n}(z, t, \Delta)\right]\right\} \psi(z) \mathrm{d} z .
\end{aligned}
$$

If we now require the following conditions suggested by a generalized random walk:

$$
\begin{aligned}
& \lim _{\Delta \rightarrow 0} \frac{A_{1}(z, t, \Delta)}{\Delta}=A(z, t) \\
& \lim _{\Delta \rightarrow 0} \frac{A_{2}(z, t, \Delta)}{\Delta}=B(z, t) \\
& \lim _{\Delta \rightarrow 0} \frac{A_{n}(z, t, \Delta)}{\Delta}=0(\Delta) \quad n \geqslant 3 .
\end{aligned}
$$

The following relations hold for arbitrary smooth $\psi(y)$ :

$$
\frac{\partial \pi(y, t)}{\partial t}=-a(y, t) \pi(y, t)-c(y, t) \frac{\partial \pi(y, t)}{\partial y}+\frac{1}{2} b(y, t) \frac{\partial^{2} \pi(y, t)}{\partial y^{2}}
$$

where $a, b, c$ are related to $A, B, C$ by simple relations. If we compare it with the Schrödinger equation

$$
\text { i } \frac{\partial}{\partial t} \psi=-\frac{\hbar^{2}}{2 m} \nabla^{2} \psi
$$

we get the correspondence

$$
\begin{aligned}
& \frac{\hbar^{2}}{2} b(y, t)=-\frac{\hbar^{2}}{2 m} . \\
& c(y, t)=a(y, t)=0 .
\end{aligned}
$$

On the other hand, the Schrödinger equation with an external scalar potential $\phi(x)$ and vector potential $A(z)$ given by

$$
\mathrm{i} \hbar \frac{\partial}{\partial t} \psi=\left\{-\frac{\hbar^{2}}{2 m}(\nabla-\mathrm{i} e A)^{2}+\phi\right\} \psi
$$


can be generated by an appropriate transformation of the dependent variable $\psi$. So the generic Chapman-Kolmogorov relations correspond to the Schrödinger equation with external scalar and vector potentials.

When the external potential $\phi$ reduces to a constant and the vector potential vanishes, the Schrödinger equation is trivially shifted from a free Schrödinger equation, and the solutions differ by the phase $\exp (-\mathrm{i} \phi t / \hbar)$ which has the power series expansion

$$
\sum_{n=0}^{\infty}(\mathrm{i} \phi t / \hbar)^{n} / n !
$$

This maybe thought of as being due to an interaction at random times with the external potential $\phi$, the number of interactions in any interval being Poisson-distributed, very much like the telephone traffic at a switchboard! This interpretation of interaction with a constant scalar potential is even more appropriate at the present juncture where particle theories consider the mass matrix of leptons to be the broken symmetry vacuum expectation value of a trilinear Yukawa coupling with one or more scalar Higgs fields.

Gaveau et al (1984) have recalled attention to this circumstance for the relativistic electron by treating the kinetic mass as a scalar potential coupling two chiral fields of opposite chirality. Each chiral field obeys one of the Weyl neutrino equations. The corresponding (perturbed) solutions maybe identified with particles travelling with the velocity of light but mutual flips at a constant mean rate proportional to mass. The successive approximations correspond to the power series expansion of $\exp (\operatorname{im} \beta t / \hbar)$ where $\beta$ is the Dirac matrix which is off-diagonal in the chiral representation of the Dirac spinors (Bose et al 1959).

The doubling of the components could have been incorporated from the beginning in the Chapman-Kolmogorov equations and the complex amplitudes in terms of a chirality index. A priori such a decomposition should be there for all relativistic equations (Foldy 1951) since the Lorentz group over the field of complex numbers splits into the direct product of two chiral rotation groups locally. However, a spin $s>\frac{1}{2}$ particle of finite mass has $2(2 s+1)$ components while the corresponding massless particles have $2 \times 2$ components only. Thus to get relativistic invariance for $s \geqslant 1$ we must include degenerate particles of zero mass of spins $s, s-1, \cdots, 0$ for integer spins and $s, s-1, \cdots, \frac{1}{2}$ for half-integer spins. So this random walk derivation for relativistic particles is not very natural for spins greater than $\frac{1}{2}$. However, we can, following Foldy, write the equations in the form

$$
\mathrm{i} \frac{\partial}{\partial t} u_{ \pm}=\omega_{ \pm} u_{ \pm}+m \rho
$$

where $u_{ \pm}$are the positive and negative chiral components with $(2 s+1)$ components on which act the spin matrices $S_{ \pm}$with the generalized mass matrix $\rho$ which intertwines the positive and negative chiral components; and can interpret $m \rho$ as generating a random-jump sequence.

\section{Hamiltonian evolutions and decay of correlations}

The Hamiltonian evolution kernel is defined not only for positive but also for negative times. These kernels satisfy the unitarity property

$$
\int\left(f_{t, t_{0}}(x \mid y)\right)^{*} \cdot\left(f_{t, t_{0}}\left(x^{\prime} \mid y\right)\right) \mathrm{d} y=\delta\left(x-x^{\prime}\right)
$$


for all $x, x^{\prime}, t, t_{0}$. Consequently the complex amplitudes preserve their norm:

$\int \psi^{\dagger}(x) \psi(x) \mathbf{d} x=\iint\left(f_{t, t_{0}}(x \mid y) \psi(y) \mathrm{d} y\right)^{*} \int f_{t, t_{0}}\left(x \mid y^{\prime} \psi\left(y^{\prime}\right) \mathrm{d} y^{\prime}\right) \mathrm{d} x$.

Hence the $(\mathrm{mod})^{2}$ norm is preserved. Furthermore, since this is a non-negative measure, it is a standard measure which can be superimposed on the complex measures.

Despite the time invariance of the quadratic norm, the complex amplitudes mapped by these kernels can exhibit (approximate) decay properties and thus provide a framework for describing metastable excitations. To see how a decay can be obtained for a system in which the evolution operator has unimodular spectrum $\left\{\mathrm{e}^{-\mathrm{i} \omega\left(t-t_{0}\right)}\right\}$ (the Hamiltonian has real spectrum $\{-\infty<\omega<\infty\}$ ), we consider the standard example of a state which is a complex linear combination of (improper) eigenstates of the continuous spectrum with a spectral density. We write

$$
\begin{aligned}
& \psi(x)=\int \mathrm{d} \omega g(\omega) \psi_{\omega}(x) \\
& \int f_{t, t_{0}}(x \mid y) \psi(y) \mathrm{d} y=\int \mathrm{d} \omega g(\omega) \mathrm{e}^{-\mathrm{i}\left(z t-t_{0}\right) \omega} \psi_{\omega}(x)
\end{aligned}
$$

and

$$
g(\omega)=\pi^{-1 / 2}\left\{\left(\omega-\omega_{0}\right)-\frac{1}{2} \mathrm{i} \Gamma\right\}^{-1}
$$

Then

$$
\psi(x, t) \equiv \int f_{t}(x \mid y) \psi(y) \mathrm{d} y
$$

satisfies

$$
\begin{aligned}
A\left(t, t^{\prime}\right) & =\int \psi^{*}(x, t) \psi\left(x, t^{\prime}\right) \mathrm{d} x \\
& =\mathrm{e}^{-\frac{1}{2}|\Gamma|\left|-t^{\prime}\right|} \int \psi^{*}(x, 0) \psi(x, 0) \mathrm{d} x=\mathrm{e}^{-\frac{1}{2}|\Gamma| t-t^{\prime} \mid} \cdot 1 .
\end{aligned}
$$

This result can be easily obtained by a contour integration. The open contour from $-\infty$ to $+\infty$ along the real axis can be closed by adding either the semi-circle at infinity in the lower half-plane for $t-t^{\prime}>0$ or in the upper half-plane for $t-t^{\prime}<0$. In either case the integrand is

$$
|g(\omega)|^{2}=\pi^{-1}\left\{\left(\omega-\omega_{0}\right)^{2}+\frac{1}{4} \Gamma^{2}\right\} .
$$

The (mod) $)^{2}$ non-negative probability measure is then monotonically decreasing:

$$
P\left(t-t^{\prime}\right)=\left|A\left(t-t^{\prime}\right)\right|^{2}=\mathrm{e}^{-|\Gamma|\left|t-t^{\prime}\right|} .
$$

Without loss of generality we may choose $\Gamma>0$ so that we may write

$$
\begin{aligned}
& A\left(t, t^{\prime}\right)=\mathrm{e}^{-\frac{1}{2} \Gamma\left|t-t^{\prime}\right|} \\
& P\left(t, t^{\prime}\right)=\mathrm{e}^{-\Gamma\left|t-t^{\prime}\right|} .
\end{aligned}
$$

Note that the wave amplitude still has a constant $(\bmod )^{2}$ norm:

$$
\int \psi^{*}(x, t) \psi(x, t) \mathrm{d} x=\int \psi^{*}(z) \psi(x) \mathrm{d} x .
$$


In obtaining this explicit form we had a spectral density $g(\omega)$ which was analytic in the interval $-\infty<\omega<\infty$. If the Hamiltonian had a spectral density of this form but restricted to $0<\omega<\infty$ we would not obtain this form exactly, but for $\Gamma>0, t-t^{\prime}>0$ the expression for $A(t)$ becomes

$$
A\left(t, t^{\prime}\right)=\frac{1}{\pi} \int_{0}^{\infty}\left\{\left(\omega-\omega_{0}\right)^{2}+\frac{1}{4} \Gamma^{2}\right\}^{-1} \mathrm{e}^{-\mathrm{i} \omega\left(t-t^{\prime}\right)-\frac{1}{2} \Gamma\left(t-t^{\prime}\right)} \mathrm{d} \omega .
$$

By adding the quarter-circle at infinity in the lower right hand side we can rewrite

$$
A\left(t, t^{\prime}\right)=A\left(t-t^{\prime}\right)=\mathrm{e}^{-\frac{1}{2} \Gamma\left(t-t^{\prime}\right)}+\frac{\mathrm{i}}{\pi} \int_{0}^{\infty} d \nu\left\{\left(\nu+\mathrm{i} \omega_{0}\right)^{2}-\frac{1}{4} \Gamma^{2}\right\}^{-1} \mathrm{e}^{-v\left(t-t^{\prime}\right)} \mathrm{d} v
$$

which can be approximated by the first term for most values of $t-t^{\prime}$ except when it is very large $\left(\gtrsim 100 \Gamma^{-1}\right.$ ) or very small. For very large values the second term contributes an inverse power term which dominates the exponential, while for very short time $A(t)$ has a vanishing derivative at $t=0$.

The crucial property involved in all these evaluations is the fact that the function $g(\omega)$ is the boundary value of an analytic function of $\omega$. The contour deformations in the actual evaluation maybe related to the generic possibility of extending the complex measure to complex values of the frequency space analytically. We therefore turn to the study of analytic complex measures.

\section{Analytic complex measures}

Let $\psi(\omega)$ be a complex measure on a measurable space $\Omega=\{\omega\}$. For fixing our ideas we choose $\Omega$ to be one or more copies of the real line or segments thereof. Then any complex measure (whose (modulus) ${ }^{2}$ norm is integrable over $\Omega$ ) may be approximated in probability by analytic complex measures. Thus analytic complex measures are dense in the (linear) space of complex measures. We have nested sequences of analytic complex measures with increasing domains of analyticity. (Sudarshan et al 1978, Sudarshan and Chiu 1993, Chiu et al 1992).

Given any analytic complex measure $\psi(\omega)$ we may associate it with a measure defined over the extended space $\Xi=\{\zeta\}$

$$
\psi(\omega) \leftrightarrow \psi(\zeta)
$$

The $(\bmod )^{2}$ measure can also be extended by the formula

$$
|\psi(\omega)|^{2} \rightarrow \psi^{*}\left(\zeta^{*}\right) \psi(\zeta \equiv a(\zeta)
$$

The right hand side is an analytic function in $\Xi$ and therefore the survival amplitude

$$
A(t)=\int \psi^{*}\left(\zeta^{*}\right) \psi(\zeta) \mathrm{e}^{-\mathrm{i} \zeta t} \mathrm{~d} \zeta=\int a(\zeta) \mathrm{e}^{-\mathrm{i} \zeta t} \mathrm{~d} \zeta
$$

may be evaluated by analytic continuation of the integrand from $\Omega$ to $\Xi$ and deformation of the contour.

The generic $\psi(\zeta)$ leads to $a(\zeta)$ being the boundary value of an analytic function in $\Xi$ but does not specify the domain of analyticity. Under rather general conditions one can express $a(\zeta)$ as the sum of two functions $a_{+}(\zeta)$ and $a_{-}(\zeta)$ which are analytic in the lower and the upper half-planes, respectively. This is displayed by the formulae

$$
a_{ \pm}(\zeta)=\frac{\mp 1}{2 \pi \mathrm{i}} \int \mathrm{d} \omega a(\omega)(\zeta-\omega \pm \mathrm{i} \epsilon)^{-1}
$$


where $\epsilon$ is a positive infinitesimal. Then

$$
A_{ \pm}(t)=\int a_{ \pm}(\zeta) \mathrm{e}^{-\mathrm{i} \zeta t} \mathrm{~d} \zeta
$$

vanishes for one sign of $t(t>0)$ but can be evaluated in terms of the contributions from the singularities of $a_{ \pm}(\zeta)$ in the lower/upper half-plane. If the singularities are only poles these contributions would be the residues at these poles. The pole contributions would be decaying exponentials.

\section{Summary and conclusion}

Motivated by the need to enlarge the theory of stochastic processes, we have dealt with an indexed family of complex measures and studied the salient features of generalized stochastic processes that arise from them. We have demonstrated that such a class of generalized stochastic processes encompasses in itself complex amplitudes extensively used in the formulation of quantum mechanics. Besides we have shown that the composition law for the transformation brackets introduced by Dirac (1933) and extensively employed by Feynman for the formulation of the path integral formalism can be viewed as a consequence of the Markov property of the underlying stochastic process. The evolution equation and the operator formalism of the first quantized version are shown to be natural consequences of the Markov property. It is shown that the generalized theory of stochastic processes is viable enough to describe the generalized version of simple standard stochastic processes like the binomial process, Poisson process and most general jump processes like population process beside the celebrated diffusion process. A demonstration is also provided leading to the version of central limit theorem in which a sum of independent and identicaliy distributed random variables converge in probability to a complex normal variable. Thus, while the complex nature of the probability measure can bring out the physical process of interference, the Markov property brings to the fore the diffractive nature of the conditional probability amplitudes. Many interesting properties like decay of correlations and the meromorphic structure of amplitudes that are normally studied on the basis of von Neumann algebras and their representations can be studied conveniently by the direct use of the properties of generalized stochastic processes, a noteworthy feature being that the Schrödinger (and Dirac 1958) equation with potentials can be brought within the framework of the FokkerPlanck equation of appropriate stochastic processes. Since a complex measurable space has a rich structure in that new positive definite measures can be defined over it, it makes itself amenable to 'observation'. Although we have not proved any limit theorem other than the central limit theorem leading to complex diffusion, it is indeed possible to establish such theorems using the ideas presented in this paper. We hope the formulae proposed in the paper can be used in other areas where interference and diffraction predominate over other additive properties.

\section{References}

Ash R B 1972 Real Analysis and Probability (New York: Academic)

Baker G A 1958 Phys. Rev. 1092198

Chiu C B, Sudarshan E C G and Bhamathi G 1992 Phys. Rev. D 463508

Bose S K, Gamba A and Sudarshan E C G 1959, Phys. Rev. 1131661

Dirac P A M 1933 Physikalisch. Zeitschrift der Sowjetunion 3 Heft (Also in

Schwinger J (ed) 1958 Selected Papers in Quantum Electodynamics (London: Dover))

-1958 Principles of Quantum Mechanics 4th edition (Oxford: Oxford University Press) 
Erber T, Hammerling P, Hockney G, Porrati M and Putterman S 1989 Ann. Phys. 190254

Feynman R P 1948 Rev. Mod. Phys. 20267

Gudder S P 1990 Quantum Probability (New York: Academic)

Gaveau B, Jacobson T, Kac M and Schulman L S 1984 Phys. Rev. Leti. 534419

Gabor D 1946 J. IEE 93429

Foldy L L 1951 Phys. Rev. 84168

Halmos 1950 Measure Theory (Princeton, NJ: Van Nostrand)

Jakeman E and Shepherd T J 1987 J. Opt. Soc. Am. B 41860

Jakeman E 1980 Opt. Acta 27735

Jordan T F and Sudarshan E C G 1961 Rev. Mod. Phys. 33515

Moyal J E 1949 Proc. Camb. Phil. Soc. 4599

Pitt H R 1963 Integration, Measure and Probability (London: Oliver and Boyd)

Ramakrishnan A, Vasandervan R and Rajagopal P 1960 J. Math. Anal. Appl. 1145

Shepherd T J 1981 Opt. Acta 28 56T

Srinivasan S K 1963 J. SIAM 11355

-1988 Pramana (India) 3059

1990 J. Phys. A: Math. Gen. 23 L369-73

Srinivasan S K and Sridharan V 1990 J. Phys. A: Math. Gen. A 23491

Sudarshan E C G 1969 J. Math. Phys. Sci. 3121

Sudarshan E C G and Chiu C B 1993 Phys. Rev. D 463508

Sudarshan E C G, Chiu C B and Gorini 1978 Phys. Rev. D 182914

Wigner E P 1932 Phys. Rev. 40749

Wolf E 1955 Proc. R. Soc. A 230246 